\title{
Imaging Findings and Changes in Discharged Moderate Novel Coronavirus Pneumonia Patients with Positive Nucleic Acid Retest
}

\author{
Wang Junda ${ }^{1}$, Deng xin ${ }^{2}$, Zhang xia ${ }^{2}$, Zhu Xinping ${ }^{3}$, Ke Yongchun ${ }^{3}$, Zhou Pan ${ }^{3}$, Li Zhi ${ }^{3}$ and Wang Jie ${ }^{4, *}$ \\ ${ }^{1}$ Radiology Department, Chongqing Hospital of Traditional Chinese Medicine, Chongqing, China \\ ${ }^{2}$ Endocrinology Department, Chongqing Hospital of Traditional Chinese Medicine, Chongqing, China \\ ${ }^{3} \mathrm{CT}$ and MRI Department, Xiaogan First People's Hospital, Xiaogan, China \\ ${ }^{4}$ Respiratory Department, Chongqing Hospital of Traditional Chinese Medicine, Chongqing, China
}

* Corresponding author: Wang Jie, Respiratory Department, Chongqing Hospital of Traditional Chinese Medicine, Chongqing, China. Email: wangjie1898@sina.com

Received 2021 March 11; Revised 2021 July 05; Accepted 2021 September 20.

\begin{abstract}
Background: The clinical and imaging data from 52 patients with positive nucleic acid re-test during the normal NCP rehabilitation period were analyzed to compare the discharged and readmitted patients in terms of dynamic changes of computed tomography (CT) features. objectives: This study aimed to investigate the CT images of the confirmed NCP patients during the follow-up period after discharge to provide a reference for the follow-up NCP evaluation.

Methods: The clinical and imaging data belonging to 52 discharged novel coronavirus pneumonia (NCP) patients with positive nucleic acid retesting results during the follow-up period were collected for analysis from the First People's Hospital, Xiaogan, Hubei Province, China. These moderate NCP patients underwent a plain CT scan before the admission, at the time of discharge, and at re-admission due to the positive result of their COVID-19 nucleic acid retesting. The CT signs of these patients were analyzed in this study.

Results: A total of 32 (61.54\%) patients presented with 123 lesions with patchy fuzzy shadows. In addition, 24 (75\%) and 8 (25\%) patients out of the 32 patients had lesions distributed in both lungs and single lung, respectively. The CT manifestations varied in discharged moderate NCP patients whose COVID-19 nucleic acid retest was positive in the follow-up period. Most patients presented with patchy fuzzy shadows and ground-glass opacity (GGO) lesions distributed in the lower lobe of both lungs.

Conclusion: Based on the obtained results, some discharged moderate NCP patients with positive COVID-19 nucleic acid retest developed new patchy fuzzy shadows and GGO lesions. The recurrence of NCP needs to be verified once patchy fuzzy shadows and GGO lesions appear and are not absorbed during the follow-up period. This study provides a reference for the follow-up NCP evaluation.

Keywords: Computed tomography, Discharged patients, Novel coronavirus pneumonia, Positive nucleic acid retesting
\end{abstract}

\section{Background}

The symptoms of novel coronavirus pneumonia (NCP) are categorized into slight, moderate, severe, and critical, in terms of severity. The patients with slight and moderate symptoms form the largest group of NCP patients (1). A total of 62,918 cases of NPC patients have been reported cured in China as of 24:00, March 12, 2020, according to the National Health Committee (NHC) of the People's Republic of China (PRC). Effective prevention and control measures and active clinical treatment were implemented in China. Most of the cured cases presented slight and moderate symptoms. The main criteria for recovery based on the Diagnosis and Treatment Protocol for Novel Coronavirus Pneumonia (Trial Version 7) developed by NHC of the PRC, General Office (2), included normal body temperature for more than three days, significant improvement of respiratory symptoms, significant improvement of inflammation based on lung imaging, and two consecutive negative results of the respiratory pathogenic nucleic acid test (sampling every other day). However, in recent clinical practice, the result of COVID19 nucleic acid retesting of some recovered patients was positive during the rehabilitation and follow-up periods, despite the fact that they had met the recovery criteria and had been discharged from the hospital. Most of these patients were concentrated and quarantined at the isolation point. Those patients with positive nucleic acid retested were shunted to different hospitals due to the statistical limitations of the sample size in every single center. The research group was dispatched to provide medical support to the First People's Hospital, Xiaogan, Hubei Province, China, which is the second city in China with the most severe cases of NCP. In this study, clinical and imaging data from 52 patients with positive nucleic acid re-test during the normal NCP rehabilitation period were analyzed to compare the discharged and readmitted patients in terms of dynamic changes of computed tomography (CT) features. The results revealed that the CT images of the confirmed NCP patients during the follow-up period after discharge provided a reference for the follow-up NCP evaluation.

\section{Methods}

\subsection{Patients}

From March 1, 2020, to March 14, 2020, 52 moderate NCP recovered patients were readmitted to Wuhan Xiaogan First People's Hospital, China, during

Copyright (C) 2021, Author(s). This is an open-access article distributed under the terms of the Creative Commons Attribution-NonCommercial 4.0 International License (http://creativecommons.org/licenses/by-nc/4.0/) which permits copy and redistribute the material just in noncommercial usages, provided the original work is properly cited 
their rehabilitation period. These cases were retested positive for COVID-19 nucleic acid during the followup period. The inclusion criteria included the moderate symptoms of NCP during the first COVID19 infection; CT scanning for patients during hospitalization, discharge, and re-admission stages; and positive result of COVID-19 nucleic acid retest during the follow-up period. The criteria for rehabilitation discharge were confirmed jointly by the NCP expert group from the Department of Respiratory of Traditional Chinese Medicine Hospital, Chongqing, China, and Xiaogan First People's Hospital based on the Diagnosis and Treatment Protocol for Novel Coronavirus Pneumonia (Trial Version 7) developed by the NHC of the PRC, General Office. However, patients with slight, severe, or critical NCP and those with no history of plain CT scans during hospitalization, after discharge, or during the follow-up period were excluded from the study .

The study population included 28 males and 24 females aged 48-63 years, with a mean age \pm SD of $52.5 \pm 11.6$ years. Moreover, the hospitalization period and the average length of stay in the hospital were $11-21$, and 13 days, respectively. In total, 9 (17.31\%) patients out of 52 patients had underlying diseases, including hypertension $(\mathrm{n}=3)$, diabetes $(\mathrm{n}=3)$, tuberculosis $(\mathrm{n}=1)$, hepatitis $\mathrm{B}$ and $\mathrm{C}(\mathrm{n}=1)$, and a history of surgery $(n=1)$. In addition, $17(32.69 \%)$ patients showed abnormal clinical manifestations, such as cough $(\mathrm{n}=14)$, diarrhea $(\mathrm{n}=2)$, and chest tightness $(\mathrm{n}=1)$. Furthermore, $10(19.23 \%)$ patients exhibited abnormal laboratory results, including decreased white blood cells $(n=2)$, decreased lymphocyte ratio $(\mathrm{n}=2)$, and decreased lymph count $(\mathrm{n}=6)$. Moreover, 9 (17.31\%) patients had abnormal coagulation. The study was approved by the Ethics Committee of Chongqing Hospital of Traditional Chinese Medicine, Chongqing, China, and informed written consent was obtained from the patients or their families.

\subsection{CT examination}

GE 64-row 128-slice spiral CT (U.S.A, Light Speed CT) was utilized for High-Resolution Continuous Scanning from the apex to the bottom of the lung. The scanning conditions were $120 \mathrm{kV}, 100$ $\mathrm{mAs}$, with layer thickness and layer spacing of 4.8 $\mathrm{mm}$. Lung window images were reconstructed using a lung algorithm with a window width and a window level of $1500 \mathrm{Hu}$ and $600 \mathrm{Hu}$, respectively. Mediastinal window images were reconstructed using a standard algorithm with a window width and a window level of $400 \mathrm{Hu}$ and $35 \mathrm{Hu}$, respectively. Multiplanar reconstruction (reconstruction layer thickness of $0.625 \mathrm{~mm}$ ), volume reconstruction, and other postprocessing were performed on thin-layer lung images of all patients using the processing workstation before transferring to Picture Archiving and Communication Systems (PACS).

\subsection{Clinical analysis}

All clinical data were jointly evaluated by the Department of Respiratory of Traditional Chinese Medicine Hospital, Chongqing, China, and First People's Hospital, Xiaogan, China. The observation criteria were developed based on the Experts Recommendations from the Chinese Medical Association Radiology Branch (First Edition) (3). Two attending physicians (with 7 and 12 years of working experience, respectively) recorded and analyzed the data blindly and independently. Clinical indicators included gender, age, the course of the disease from the first diagnosis of NCP to rehabilitation and discharge, previous history of underlying diseases, clinical symptoms at re-admission, and laboratory indicators .

All study participants underwent a CT scan before admission, at discharge, during rehabilitation, and at re-admission (after discharge). Image analysis focused on lesion size, distribution, morphology, presence of pleural effusion, and lymphadenopathy. The CT findings were compared at the three-time points, and the relationship between imaging findings and clinical symptoms was investigated. The investigation included the observation of the location and distribution of single and multiple lesions (the number of lesions is no longer counted but is referred to as multiple lesions) in either one or both lung lobes. The lesion morphology was determined by patchy shadows (patchy shadows under the lung window, blurred borders with blurred stripes), ground-glass opacity (GGO), the density of lesions (reduced local transparency of the lung tissue, light shadows, and uneven density through which the structure of the bronchial vascular bundle lungs can be seen and is generally only displayed on the lung window but not on the mediastinal window), fibrous lesions (clear strip-shaped high-density shadows below the lung window) (4-6). No obvious absorption of patchy foci was defined if the CT performance was the same at discharge and re-admission due to positive results of nucleic acid retesting. The narrowing of the lesion focus was defined as a 1-3 mm reduction in lesion maximum diameter measured by both attending physicians, and complete absorption was defined as complete disappearance of the lesions along with positive nucleic acid retesting result.

\section{Results}

3.1. Chest high-resolution computed tomography scan after positive nucleic acid retesting during the rehabilitation period after discharge

Chest high-resolution computed tomography (HRCT) axial scan of a representative discharged patient during rehabilitation showed patchy inflammation in the upper and lower lobes of the left lung (arrow), distributing around the lungs with 
different flocculent-like changes (Figure 1A). Furthermore, chest HRCT axial scan of the patient with positive nucleic acid test showed that the patchy inflammation of the left lung tongue segment was clearly absorbed (arrow), and the patchy fuzzy lesions in the lower lobe of the left lung were newly formed (arrow) within one week after discharge. The peripheral lesions of the left upper lobe of the lung were clearly absorbed, the density was slightly increased, and the edges were clear. These were small pieces with peripheral fibrous changes, and the pleura was not stretched (Figure 1B).
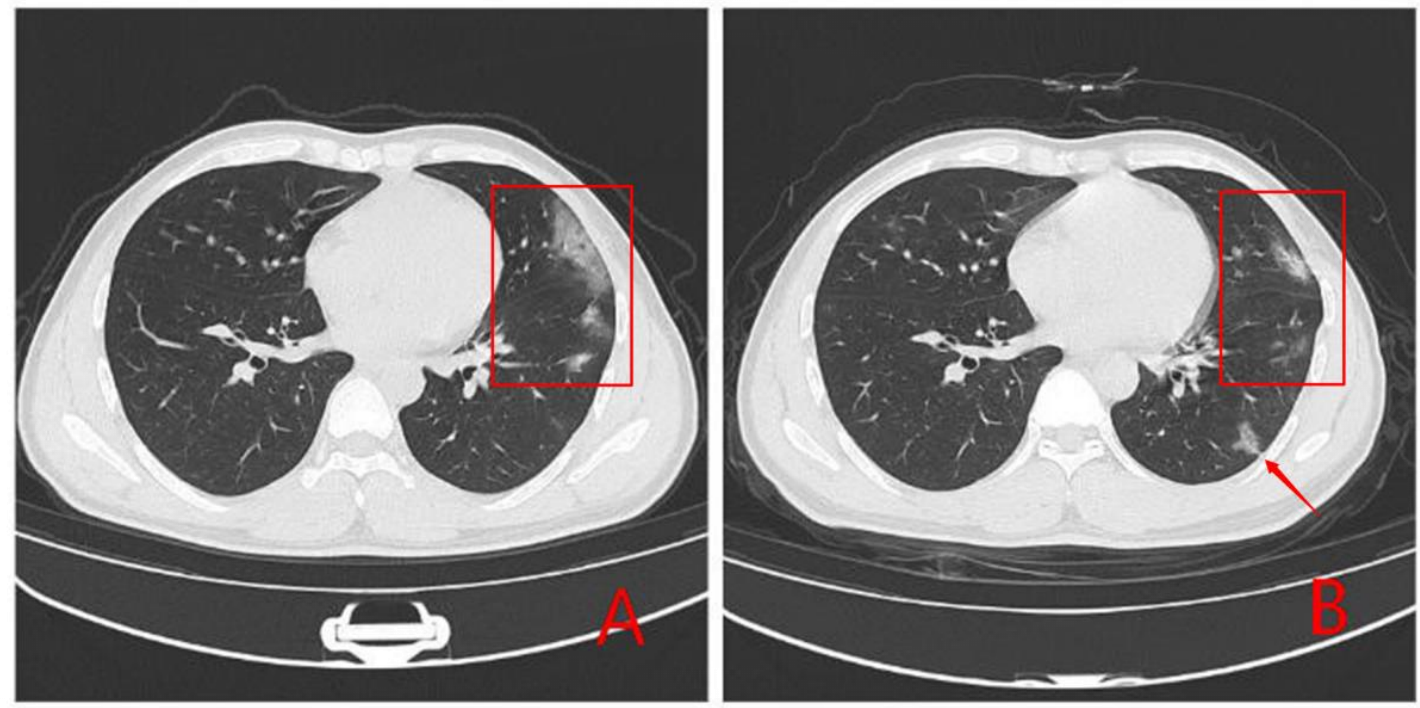

Figure 1. The high-resolution computed tomography images of the obvious absorption of patchy inflammation and new patchy inflammation in the lung.

The chest HRCT axial scan of another representative discharged patient during rehabilitation showed patchy inflammation in the lower lobe of both lungs (arrow), and the lower lobe of both lungs showed a strip of slightly high-density shadow, with local interstitial changes and no pleural effusion (Figure 2A). In another patient with positive nucleic acid retesting, the chest HRCT axial scan showed that the patchy bilateral inflammation in the lungs was not absorbed (arrow) within 1-2 weeks after discharge. There was no visible absorption of flaky, slightly dense density in the lower lobe of lungs, no new lesions, and no pleural effusion (Figure 2B).

Moreover, the chest HRCT axial scan of another representative discharged patient during the rehabilitation period showed a ground-glass opacity (GGO) lesion in the lower left lobe (arrow), a flocculent fuzzy shadow with clear edges in the left inferior lobe of the left lung, and changes in the bronchial passage and
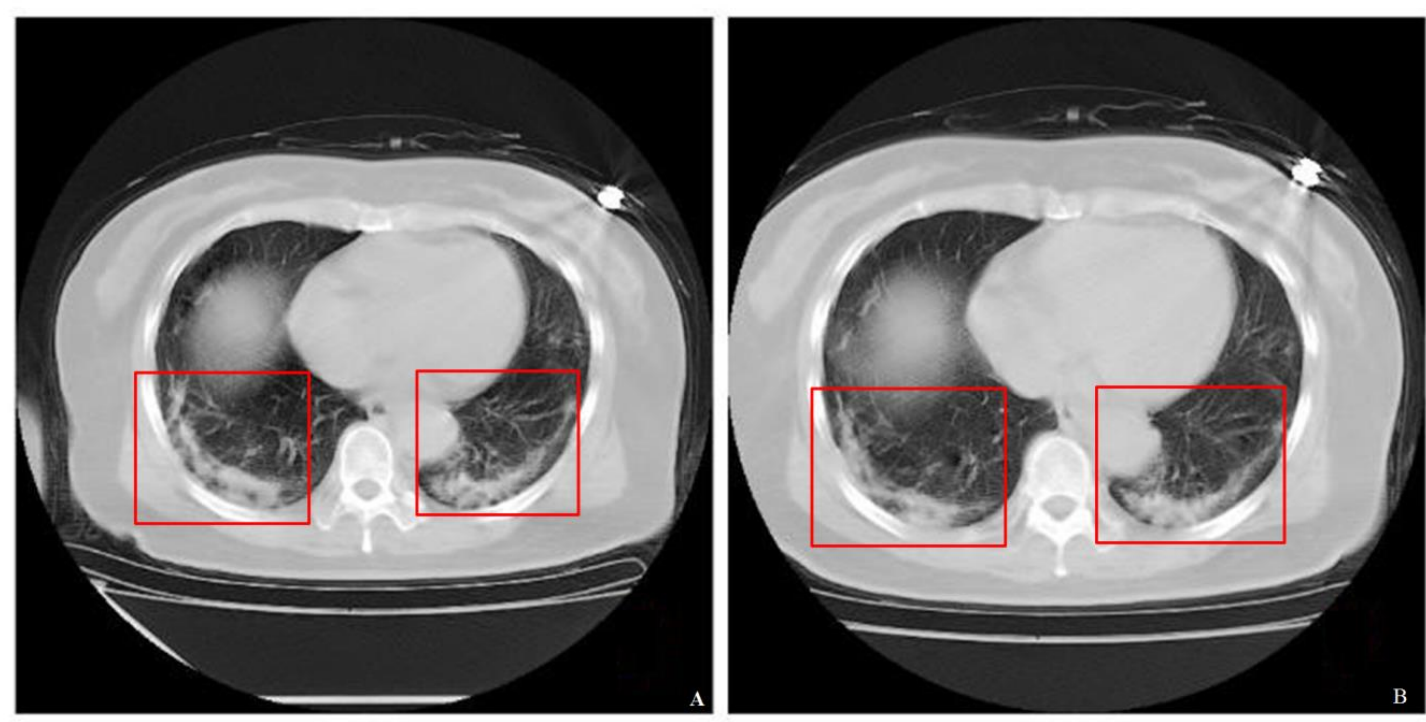

Figure 2. The image shows that the patchy inflammation in the lungs was not absorbed. A. Chest high resolution computed tomography axial scan of a discharged patient during rehabilitation B. Chest high resolution computed tomography axial scan of the patient with positive nucleic acid retesting. 
pleural traction (Figure 3A). A Chest HRCT axial scan of another patient with positive nucleic acid retesting results showed that the GGO lesion in the lower left lobe was absorbed (arrow) within 1-2 weeks after discharge at re-admission. Moreover, the flocculent fuzzy shadow of the left inferior lobe was reduced, the density became lighter, the fringe appeared on edge, and pleural traction signs disappeared (Figure 3B).

The chest HRCT axial scan of a discharged patient during the rehabilitation period revealed GGO lesions in the lower lobe of both lungs (arrow). The upper lobe of the right lung along with the bronchi had patchy fuzzy inflammation. In addition, the upper and lower lobes of the right lung showed a slightly high-density shadow with a flocculent edge along with the bronchial vascular bundles (Figure 4A). Furthermore, a flocculent fuzzy shadow with bronchial crossing changes was observable in the lower lobe of both lungs (Figure 4A). Chest HRCT axial scan of another patient with positive nucleic acid retesting results showed that the GGO lesion in the lower left lobe was not absorbed in re-admission within one week after discharge (arrow), and the GGO lesion in the lower right lobe and the upper leafy patchy fuzzy inflammation were
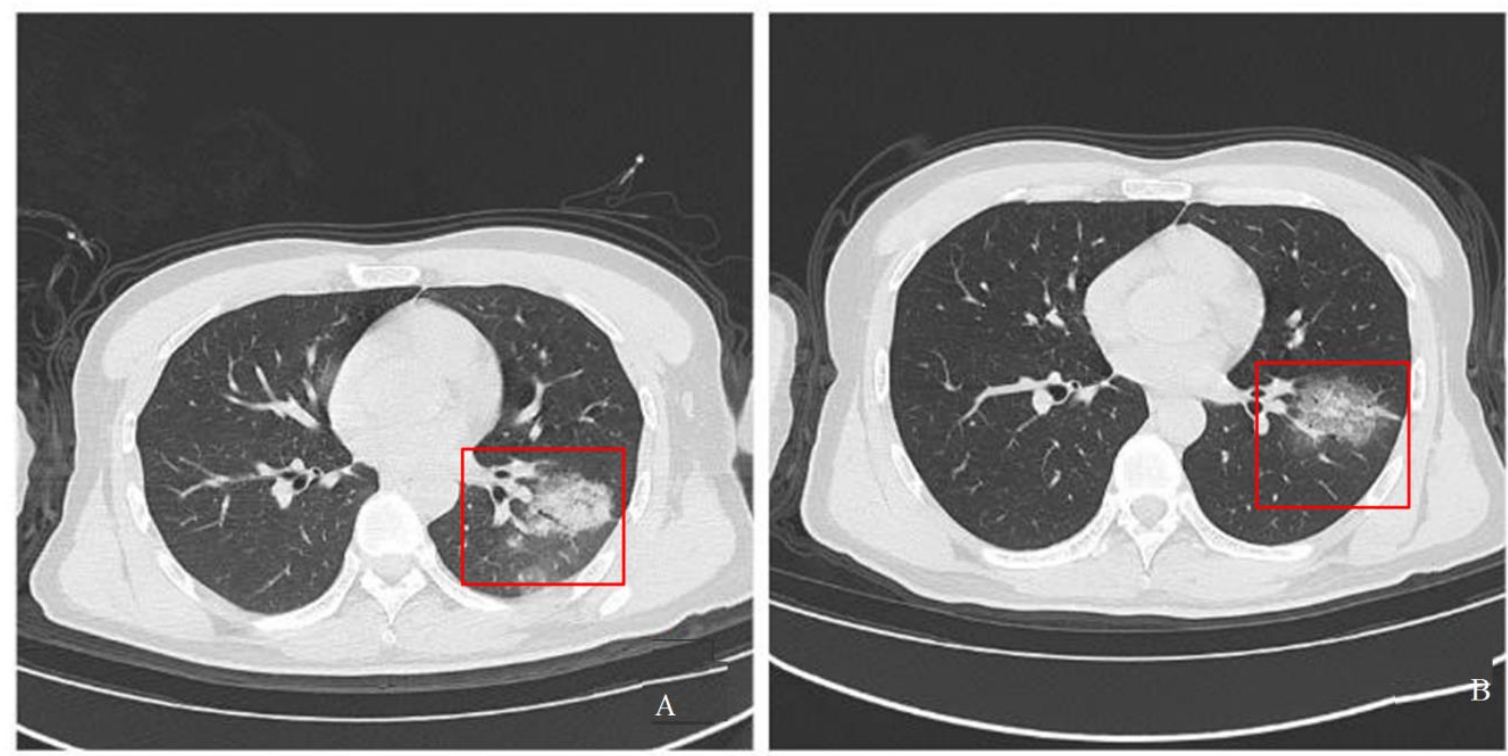

Figure 3. Obvious absorption of ground-glass opacity lesion in the lung. A. Chest high resolution computed tomography axial scan of a patient at discharge during rehabilitation B. Chest high resolution computed tomography axial scan of the patient with positive nucleic acid retesting.
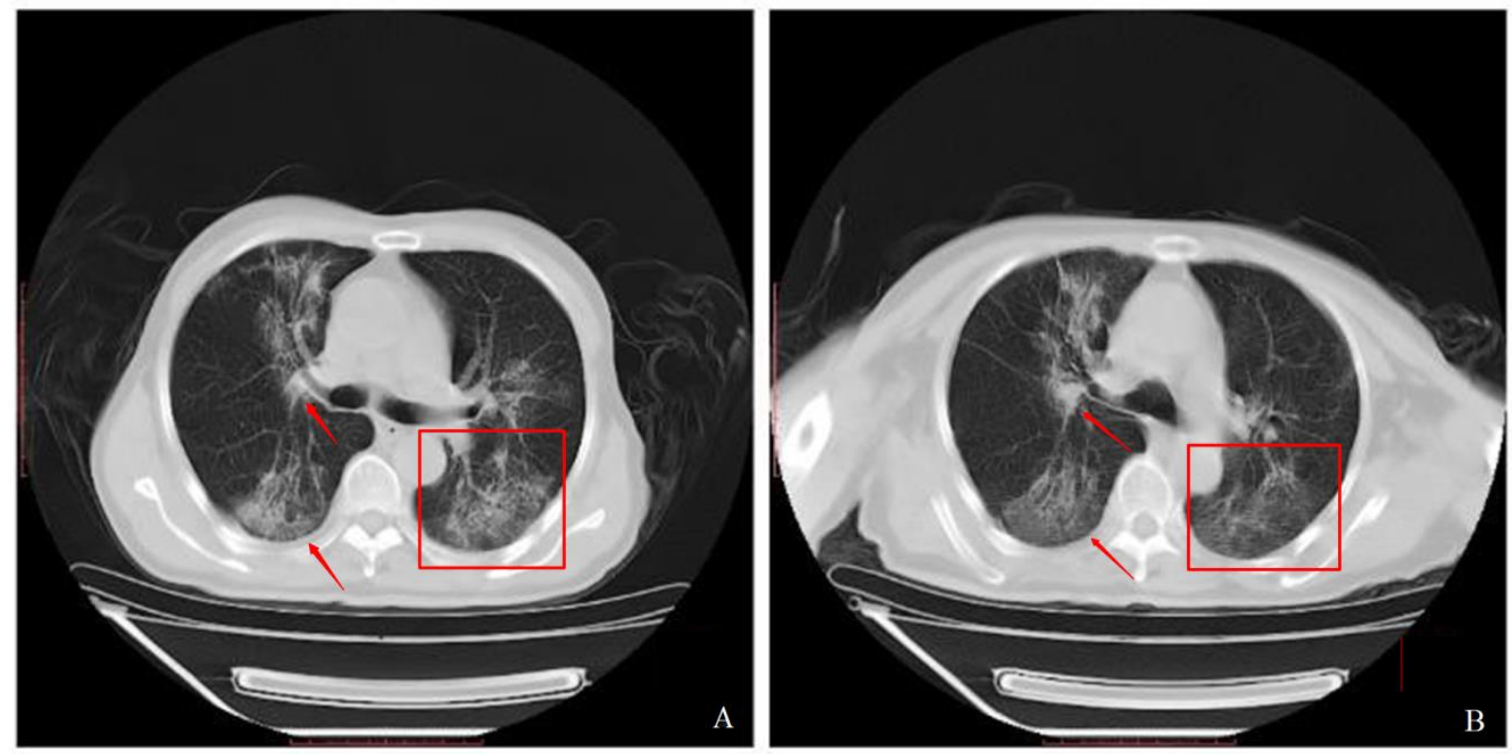

Figure 4. Ground-glass opacity in the lung was not absorbed. A. Chest high resolution computed tomography axial scan of a patient at discharge during rehabilitation B. Chest high resolution computed tomography axial scan of the patient with positive nucleic acid retesting. 
slightly absorbed (arrows). The flocculent fuzzy shadows in the upper and lower lobes of the right lung along with the bronchial vascular bundles were significantly reduced, the surrounding striated foci were changed, the density of the flocculent blurred shadows in the lower inferior lobe of the left lungs became lighter, the range was reduced, and the edges were more obvious than before (Figure 4B).

The patients with two consecutive negative nucleic acid tests were discharged from the hospital after symptomatic treatment. A CT scan at the time of discharge revealed multiple GGOs along with the bronchial vascular bundles (Figure 5A). The scope of the lesion was small, the boundary was clear, and the bronchial vascular bundle (arrow) could be observed in some lesions (Figure 5A). A thoracic HRCT axial scan was conducted at re-admission on patients whose nucleic acid re-examination was positive after one week of quarantine. Moreover, a re-examination CT scan showed visible absorption of GGO in both lungs, lightening, narrowed scope, and fiber cord lesions around the remaining lesions (arrow) (Figure 5B).

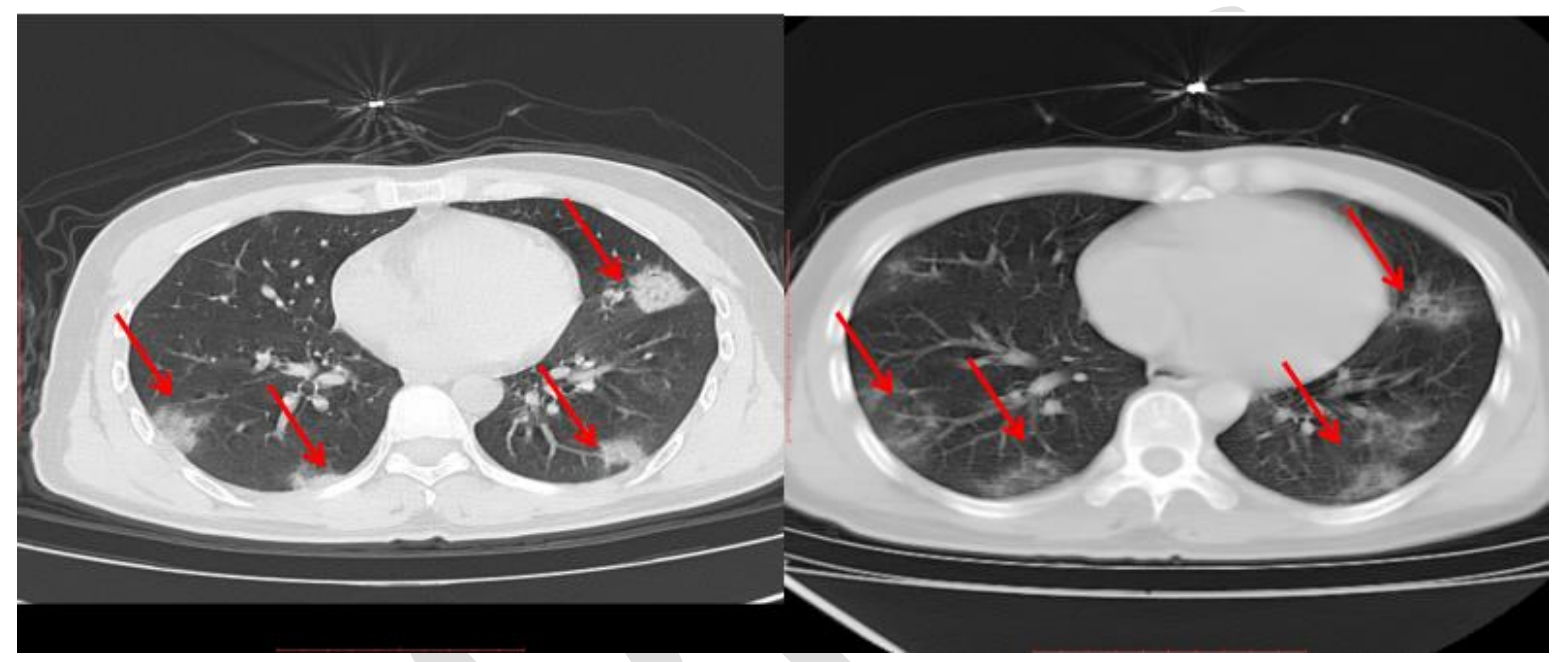

Figure 5. Ground-glass opacity is absorbed in the lungs. A. Chest high resolution computed tomography axial scan at the time of discharge and after symptomatic treatment. B. Thoracic high resolution computed tomography axial scan at re-admission following positive nucleic acid re-examination.

\subsection{Computed tomography findings of patients with} positive nucleic acid retesting results during moderate NCP rehabilitation and post-discharge follow-up

Out of the 52 patients with positive nucleic acid retest during the NCP rehabilitation period postdischarge, 32 (61.54\%) patients presented with 123 lesions with patchy blurry shadows. The lesions in 24 $(75 \%)$ and $8(25.00 \%)$ patients were bilaterally and laterally distributed, respectively. Out of the 123 lesions, 98 (79.68\%), 11 (8.94\%), and 14 (11.38\%) lesions were distributed in the lower lobes of the two lungs, the middle lobe of the right lung, and in the upper lobe of the lungs, respectively. In addition, 12 (23.08\%) patients presented with 21 GGO lesions. Out of these 12 patients, bilateral and unilateral lung lobe distribution were observed in 7 (58.33\%) and 5
(41.67\%) patients, respectively. In addition, 14 (66.67\%), 2 (9.53\%), and 5 (23.80\%) GGO lesions out of the $21 \mathrm{GGO}$ lesions were distributed in the lower lobe of both lungs, the right lung, and in the upper lobe of the lungs, respectively.

Furthermore, a total of 18 fibrous cord bands were found in $5(9.62 \%)$ patients. Fibrous cord bands in $2(40 \%)$ patients were distributed in both lungs and in $3(60 \%)$ patients had a unilateral lung lobe distribution. In addition, 10 (55.56\%), 4 $(22.22 \%)$, and $4(22.22 \%)$ fibrous bands out of the 18 fibrous bands were distributed in the lower lobe of the lung, in the right lung, and in the upper lobe of the lung, respectively. Eventually, 3 (5.76\%) patients showed no abnormal changes in either lung (Table 1).

\begin{tabular}{|c|c|c|c|}
\hline & Patchy blur & Ground-glass opacity lesions & Fibrous bands \\
\hline Both lungs & $24(75.00 \%)$ & $7(58.33 \%)$ & $2(40.00 \%)$ \\
\hline Single lung lobe & $8(25.00 \%)$ & $5(41.67 \%)$ & $3(60.00 \%)$ \\
\hline Lower lobe of both lungs & $98(79.68 \%)$ & $14(66.67 \%)$ & $10(55.56 \%)$ \\
\hline Middle lobe of right lung & $11(8.94 \%)$ & $2(9.53 \%)$ & $4(22.22 \%)$ \\
\hline Upper lobe of both lungs & $14(11.38 \%)$ & $5(23.80 \%)$ & $4(22.22 \%)$ \\
\hline
\end{tabular}


3.3. Comparison of CT findings at discharge, during rehabilitation, and at re-admission due to positive nucleic acid retesting results

Within one week after discharge from the hospital, 47 (69.12\%), 17 (25.00\%), and 4 (5.88\%) patchy legions out of the 68 patchy blurred shadows of patients $(\mathrm{N}=31)$ with positive nucleic acid retest were not absorbed, slightly narrowed, and entirely absorbed, respectively. Moreover, six new patchy blurry shadows were observed. In the same lie, out of the 15 GGO lesions, 10 (66.67\%), 4 (26.67\%), and 1 (6.66\%) lesions were not absorbed, slightly narrowed, and completely absorbed, respectively. Moreover, three new GGO lesions had appeared. However, no changes were observed in 11 fibrous bands (Figures 1, 4, 5).

It should be noted that 55 patchy blurred shadows were observed in 21 patients with positive nucleic acid re-test results within 1-2 weeks after discharge, out of which, 33 (60.00\%), 16 (29.09\%), and 6 (10.91\%) patchy lesions were not absorbed, slightly narrowed, and entirely absorbed, respectively. Moreover, three new patchy lesions appeared. Out of the 6 GGO lesions, 4 (66.67\%), 1 (16.66\%), and 1 (16.66\%) GGO lesions were not obviously absorbed, slightly narrowed, and completely absorbed. Furthermore, two new GGO lesions had been appeared (Figures 2, 3). No changes were observed in the seven cable lesions. In addition, seven patients had pleural effusion at discharge from the NCP rehabilitation period. A noticeable absorption change (absorption rate 100\%) was observed in these seven patients when the nucleic acid was retested (Table 2).

\begin{tabular}{|c|c|c|c|c|c|c|c|c|c|}
\hline & \multicolumn{4}{|c|}{ Patchy blur } & \multicolumn{4}{|c|}{ GGO lesions } & \multirow{2}{*}{$\begin{array}{c}\begin{array}{c}\text { Fibrous } \\
\text { bands }\end{array} \\
\begin{array}{c}\text { No } \\
\text { change }\end{array}\end{array}$} \\
\hline & $\begin{array}{l}\text { No obvious } \\
\text { absorption }\end{array}$ & $\begin{array}{l}\text { Range was } \\
\text { narrowed }\end{array}$ & $\begin{array}{c}\text { Complete } \\
\text { absorption }\end{array}$ & $\begin{array}{c}\text { Newly } \\
\text { appeared } \\
\text { blur }\end{array}$ & $\begin{array}{l}\text { No obvious } \\
\text { absorption }\end{array}$ & $\begin{array}{l}\text { Range was } \\
\text { narrowed }\end{array}$ & $\begin{array}{c}\text { Complete } \\
\text { absorption }\end{array}$ & $\begin{array}{c}\text { New } \\
\text { appeared } \\
\text { blur }\end{array}$ & \\
\hline $\begin{array}{l}\text { Positive nucleic } \\
\text { acid retesting } \\
\text { results within } 1 \\
\text { week after } \\
\text { discharge }\end{array}$ & $\begin{array}{c}47 \\
(69.12 \%)\end{array}$ & $17(25.00 \%)$ & $4(5.88 \%)$ & 6 & $\begin{array}{c}10 \\
(66.67 \%)\end{array}$ & $4(26.67 \%)$ & $1(6.66 \%)$ & 3 & 11 \\
\hline $\begin{array}{l}\text { Positive nucleic } \\
\text { acid retesting } \\
\text { results within } \\
1-2 \text { weeks after } \\
\text { discharge }\end{array}$ & $\begin{array}{c}33 \\
(60.00 \%)\end{array}$ & $16(29.09 \%)$ & $6(10.91 \%)$ & 3 & $4(66.67 \%)$ & $1(16.66 \%)$ & $\begin{array}{c}1 \\
(16.66 \%)\end{array}$ & 2 & 7 \\
\hline
\end{tabular}

\subsection{Interpretation}

The CT manifestations varied in discharged moderate NCP patients with positive nucleic acid retest during the follow-up period and mainly included patchy fuzzy shadows and GGO lesions distributed in the lower lobe of both lungs. The recurrence of NCP needed to be detected through the nucleic acid test once patchy fuzzy shadows and GGO lesions showed no signs of obvious absorption during the follow-up period. Some discharged moderate NCP patients who had positive nucleic acid retest during the follow-up period developed new patchy fuzzy shadows and GGO lesions. It is suggested that the patients should be closely monitored with CT to find new lesions during the follow-up period.

\section{Discussion}

Coronavirus disease 2019 (COVID-19) caused by severe acute respiratory syndrome coronavirus 2 (SARS-CoV-2) is responsible for the recent NCP outbreak. NCP is considered a self-limiting infectious disease in the majority of patients. However, the mechanism of viral clearance and the duration of this process have not been reported. According to Diagnosis and Treatment Protocol for Novel Coronavirus Pneumonia (Trial Version 7) developed by the NHC of the PRC, General Office (2), the discharge criteria include normal body temperature for more than three days, significant improvement of respiratory symptoms, significant improvement of exudative lesions, and two consecutive negative results of the respiratory pathogenic nucleic acid test (sampling every other day). In this study, 52 patients with moderate NCP showed positive nucleic acid re-testing results with specific clinical and imaging manifestations during the follow-up period post-discharge. In total, $17.31 \%$ of these patients had underlying diseases, such as hypertension and diabetes, $32.69 \%$ showed abnormal clinical manifestations with cough as the most common symptom, and $19.23 \%$ presented abnormal laboratory parameters with decreased lymphatic counts and abnormal coagulation function as common symptoms. None of these patients had a fever or D-dimer abnormalities. Moreover, no human-to-human transmission was observed among the patients with positive nucleic acid retesting results during the follow-up period, which was indicative of a good prognosis.

The research group treated more than 500 patients with moderate NCP symptoms in the First People's Hospital, Xiaogan, China. Among the recovered patients, 52 cases retested positive for COVID-19 nucleic acid during the follow-up period post-discharge. According to the group's clinical experience and knowledge, three scenarios could 
explain this occurrence: 1) Many discharged patients were still in the self-limiting recovery stage of the disease. Therefore, although a CT scan of the lungs showed that the lesions had obviously been absorbed, the virus had remained in the bronchus or paranasal sinus cavity during the viral clearance, resulting in virus reinfection in the follow-up period. 2) The medication effectively inhibited virus replication and limited the spread of the virus, leading to negative nucleic acid testing results after treatment during hospitalization. However, once the patient was discharged from the hospital and discontinued the therapy, the virus continued to replicate in large quantities, resulting in a positive nucleic acid retest during the follow-up period (8). This hypothesis was confirmed by CT manifestations of discharged moderate NCP patients. Retrospective analysis showed that there was no complete absorption of lung lesions in some patients during hospitalization. Although clinical manifestations in these patients returned to normal, and their nucleic acid testing results turned negative, the results of the nucleic acid retesting in the follow-up period during clinical observation turned positive. 3) The efficiency of the current nucleic acid testing method is not highly reliable. The testing results of moderate NCP patients during the hospitalization were intermittently negative. In addition, the quality and sensitivity of different kits varied dramatically. The positive nucleic acid retesting results of the discharged moderate NCP patients during the follow-up period can be attributed to the uncompleted viral clearance, virus virulence, viral load, and immunity of patients. It is suggested that future studies focus on the positive nucleic acid retesting results of the discharged moderate NCP patients during the follow-up period.

Chest CT scanning plays an irreplaceable role in screening, observing, and predicting the outcome of NCP treatment. Researchers at Taizhou Hospital of Wenzhou Medical University, Wenzhou, China, compared the diagnostic sensitivity of CT imaging with that of nucleic acid testing in 51 NCP patients and found that the sensitivity of chest CT was higher than that of PT-PCR for the diagnosis of NCP $(\mathrm{P}<0.01)$ (9). Radiologists at the Second Xiangya Hospital of Central South University, Changsha, China, showed that a highly effective initial screening method for suspected NCP patients included the combination of imaging (chest CT) and nucleic acid testing $(10,11)$. Regarding the observed efficacy of NCP treatment, a multicenter study demonstrated that patients with normal chest CT might also be positive for viral nucleic acid and that the chest CT scan results depended on the time course of infection $(11,12)$. Moreover, some studies revealed particular specificity of CT signs in different stages of NCP $(12,13)$, and that CT imaging played an important role in the prediction of outcome $(6,14,16)$. However, current research on NCP outcomes mainly focused on the observation of the dynamic changes in the lungs images of patients during hospitalization (16-18), while no relevant research was performed on patients in quarantine after discharge. For this reason, the present study aimed to target discharged moderate NCP patients during the post-treatment rehabilitation and followup period. Therefore, a total of 52 discharged moderate NCP patients with positive nucleic acid retest during the rehabilitation and follow-up periods were selected for this study, and the relationship between dynamic CT changes and positive nucleic acid retest was investigated. In total, $61.54 \%$ of the patients presented patchy fuzzy shadows. It should be mentioned that in $75 \%$ and $79.68 \%$ of the patients, fuzzy shadows were distributed in both lungs, and in the lower lobe of both lungs, respectively. Moreover, $23.08 \%$ of patients were presented with GGO lesions, 58.33\% of the cases had distributions in both lungs, and $66.67 \%$ of the patients had fuzzy shadow distribution in the lower lobe of both lungs. Only $9.62 \%$ and $5.76 \%$ of the patients had fibrous stripes or standard CT, respectively. For moderate NCP patients with lesion absorption post-discharge, especially patients with positive nucleic acid retesting results, the changes were more visible. In this study, 31 patients with positive nucleic acid retesting results within one week after discharge showed 68 patchy fuzzy shadows, among them $69.12 \%$ had no obvious lesion absorption, $25 \%$ had slightly narrowed lesions, and only $5.88 \%$ had complete absorption. Moreover, $66.67 \%$ of the patients $(n=15)$ with GGO lesions showed no apparent absorption, $26.67 \%$ showed slightly narrowed lesions, and GGO lesions in only $6.66 \%$ of these patients had been completely absorbed. Out of the 55 patchy fuzzy shadows of patients $(n=21)$ with a positive nucleic acid retest within 1-2 weeks after discharge, $60 \%$ had no obvious absorption, $29.09 \%$ had slightly narrowed lesions, and only $10.91 \%$ had complete absorption. Moreover, $66.67 \%$ of the GGO lesions (n=6) had no obvious absorption and $16.66 \%$ ranged from slightly narrowed to wholly absorbed. The pleural effusion in these patients was absorbed entirely, while the remaining fibrous stripes remained unchanged. Overall, the absorption process of lung lesions in patients with moderate NCP was slow. Excluding the patients with underlying disease history and medication interference, it is speculated that the absorption of lung lesions in patients with moderate NCP might take at least two weeks.

Regarding the limitations of the present study, one can refer to the fact that this was a single-center study with a limited number of participants $(n=52)$. The sample size needs to be expanded for further observations. In addition, the study included the 
recovered patients whose COVID-19 nucleic acid retested positive, and those with negative retest results were not included in the study as negative controls. Moreover, in this study, the samples for the COVID-19 nucleic acid test were throat swabs and the viral load in the nose, bronchus or pulmonary alveoli was not investigated. Therefore, further studies need to include specimens from multiple tissues to improve test efficacy. Eventually, there was a lack of complete quantitative indicators for the lesion analysis that should be considered in future studies.

\section{Conclusion}

The absorption of lung lesions in moderate NCP discharged patients with positive nucleic acid retest was slow and required at least two weeks. In addition, there was a particular relationship between positive nucleic acid retesting and lung lesion absorption in patients. Therefore, it is necessary to monitor the changes that occur in the lung lesions of moderate NCP discharged patients carefully. Moreover, it is suggested that the nucleic acid test be performed as early as possible to identify NPC recurrence in the case of patients whose lung lesions are not entirely absorbed. With the global outbreak of the coronavirus epidemic, quantitative imaging should be combined with the clinical and imaging features to improve the diagnostic criteria, therapeutic evaluation, and prediction of outcomes. Eventually, regarding the high sensitivity of CT scanning, follow-up CT is recommended to evaluate the improvement or recurrence of the NCP.

\section{Acknowledgments}

None.

\section{Footnotes}

Authors' contributions: Jie Wang, Xinping Zhu, and Yongchun Ke determined the research topics and designed the research schemes; Junda Wang wrote the paper; Xin Deng, Xia Zhang, Pan Zhou, and Zhi Li collected the data; and Jie Wang and Yongchun Ke revised the manuscript.

Ethical Approval: This study was approved by the Ethics Committee of Chongqing Hospital of Traditional Chinese Medicine, Chongqing, China.

Funding/Support: This study was supported by Chongqing Hospital of Traditional Chinese Medicine. The Special Funding Project of Chongqing Municipal Health Commission for New Coronary Pneumonia Prevention and Control Emergency Scientific Research (No. 2020NCPZX20); Chongqing Municipal Health and Family Planning Commission Traditional Chinese Medicine Science and Technology Project (No.ZY201802025); Chongqing Natural Science Foundation Project (No.cstc2019jcyj-msxmX0555);
2019 "Xinglin Scholars" Discipline Talent Research Promotion Plan (No.YYZX2019064).

Financial Disclosure: None declared.

Conflicts of Interest: The authors declare that they have no conflict of interest regarding the publication of this study

Informed consent: Informed consent was obtained from all individual participants included in the study.

\section{References}

1. Fan J, Liu X, Shao G, Qi J, Li Y, Pan W, et al. The epidemiology of reverse transmission of COVID-19 in Gansu Province, China. Travel Med Infect Dis. 2020;37:101741. doi: 10.1016/j.tmaid.2020.101741.

2. Zeng L, Xia S, Yuan W, Yan K, Xiao F, Shao J, et al. Neonatal earlyonset infection with SARS-CoV-2 in 33 neonates born to mothers with COVID-19 in Wuhan, China. JAMA Pediatr. 2020;174(7): 722-725. doi: 10.1001/jamapediatrics.2020.0878. [PubMed: 32215598].

3. Yang W, Sirajuddin A, Zhang X, Liu G, Teng Z, Zhao S, et al. The role of imaging in 2019 novel coronavirus pneumonia (COVID19). Eur Radiol. 2020;30(9):4874-82. doi: 10.1007/s00330020-06827-4. [PubMed: 32296940].

4. Kieling C, Baker-Henningham H, Belfer M, Conti G, Ertem I, Omigbodun $\mathrm{O}$, et al. Child and adolescent mental health worldwide: evidence for action. Lancet. 2011;378(9801):1515-25. doi: 10.1016/S0140-6736(11)60827-1. [PubMed: 22008427].

5. Wu J, Wu X, Zeng W, Guo D, Fang Z, Chen L, et al. Chest CT findings in patients with coronavirus disease 2019 and its relationship with clinical features. Invest Radiol. 2020;55(5):257-61. doi: 10.1097/RLI.0000000000000670. [PubMed: 32091414].

6. Chung M, Bernheim A, Mei X, Zhang N, Huang M, Zeng X, et al. CT Imaging features of 2019 novel coronavirus (2019-nCoV). Radiology. 2020;295(1):202-7. doi: 10.1148/radiol.2020200230. [PubMed: 32017661].

7. Wang D, Hu B, Hu C, Zhu F, Liu X, Zhang J, et al. Clinical characteristics of 138 hospitalized patients with 2019 novel coronavirus-infected pneumonia in Wuhan, China. JAMA. 2020;323(11):1061-9. doi: 10.1001/jama.2020.1585. [PubMed: 32031570].

8. Xu X, Chen P, Wang J, Feng J, Zhou H, Li X, et al. Evolution of the novel coronavirus from the ongoing Wuhan outbreak and modeling of its spike protein for risk of human transmission. Sci China Life Sci. 2020;63(3):457-60. doi: 10.1007/s11427020-1637-5. [PubMed: 32009228].

9. Chen $\mathrm{N}$, Zhou $\mathrm{M}$, Dong $\mathrm{X}, \mathrm{Qu} J$, Gong $\mathrm{F}$, Han $\mathrm{Y}$, et al. Epidemiological and clinical characteristics of 99 cases of 2019 novel coronavirus pneumonia in Wuhan, China: a descriptive study. Lancet. 2020;395(10223):507-13. doi: 10.1016/S01406736(20)30211-7. [PubMed: 32007143].

10. Xie X, Zhong Z, Zhao W, Zheng C, Wang F, Liu J. Chest CT for typical coronavirus disease 2019 (COVID-19) pneumonia: relationship to negative RT-PCR testing. Radiology. 2020;296(2):E41-5. doi: 10.1148/radiol.2020200343. [PubMed: 32049601].

11. Chen J, Ying H, Liu X, Gu J, Feng R, Chen T, et al. A transfer learning based super-resolution microscopy for biopsy slice images: the joint methods perspective. IEEE/ACM Trans Comput Biol Bioinform. 2021;18(1):103-13. doi: 10.1109/TCBB.2020.2991173. [PubMed: 32356757].

12. Bernheim A, Mei X, Huang M, Yang Y, Fayad ZA, Zhang N, et al. Chest CT findings in coronavirus disease-19 (COVID-19): relationship to duration of infection. Radiology. 2020; 295(3):200463. doi: 10.1148/radiol.2020200463. [PubMed: 32077789].

13. Lei J, Li J, Li X, Qi X. CT imaging of the 2019 novel coronavirus (2019-nCoV) pneumonia. Radiology. 2020;295(1):18. doi: 10.1148/radiol.2020200236. [PubMed: 32003646].

14. Wang YS, Wang Y. A gradient-based approach for optimal plant controller co-design. 2015 American Control Conference (ACC), Chicago, IL, USA; 2015. P. 3249-54. doi: 10.1109/ACC.2015.7171833. 
15. Shi H, Han X, Zheng C. Evolution of CT manifestations in a patient recovered from 2019 novel coronavirus (2019-nCoV) pneumonia in Wuhan, China. Radiology. 2020;295(1):20. doi: 10.1148/radiol.2020200269. [PubMed: 32032497].

16. Gao H, Xu Y, Yin Y, Zhang W, Li R, Wang X. Context-aware OoS prediction with neural collaborative filtering for Internet-ofThings services. IEEE Internet Things J. 2019;7(5):4532-42. doi: 10.1109/JIOT.2019.2956827.
17. Sun P, Qie S, Liu Z, Ren J, Li K, Xi J. Clinical characteristics of hospitalized patients with SARS-CoV-2 infection: a single arm meta-analysis. J Med Virol. 2020;92(6):612-617. doi: 10.1002/jmv.25735. [PubMed: 32108351].

18. Chen P, Zhang Y, Wen Y, Guo J, Jia J, Ma Y, et al. Epidemiological and clinical characteristics of 136 cases of COVID-19 in main district of Chongqing. J Formos Med Assoc. 2020;119(7):1180-4. doi: 10.1016/j.jfma.2020.04.019. [PubMed: 32386675]. 